\title{
Universal magnetic oscillations of DC conductivity in the incoherent regime of correlated systems
}

\author{
Jakša Vučičević ${ }^{1}$ and Rok Žitko ${ }^{2,3}$ \\ ${ }^{1}$ Scientific Computing Laboratory, Center for the Study of Complex Systems, \\ Institute of Physics Belgrade, University of Belgrade, Pregrevica 118, 11080 Belgrade, Serbia \\ ${ }^{2}$ Jožef Stefan Institute, Jamova 39, SI-1000 Ljubljana, Slovenia \\ ${ }^{3}$ Faculty of Mathematics and Physics, University of Ljubljana, Jadranska 19, SI-1000 Ljubljana, Slovenia
}

(Dated: August 16, 2021)

\begin{abstract}
Using the dynamical mean field theory we investigate the magnetic field dependence of DC conductivity in the Hubbard model on the square lattice, fully taking into account the orbital effects of the field introduced via the Peierls substitution. In addition to the conventional Shubnikov-de Haas quantum oscillations, associated with the coherent cyclotron motion of quasiparticles and the presence of a well-defined Fermi surface, we find an additional oscillatory component with a higher frequency that corresponds to the total area of the Brillouin zone. These paradigm-breaking oscillations appear at elevated temperature. This finding is in excellent qualitative agreement with the recent experiments on graphene superlattices. We elucidate the key roles of the off-diagonal elements of the current vertex and the incoherence of electronic states, and explain the trends with respect to temperature and doping.
\end{abstract}

Quantum oscillations (QOs) are a fundamental phenomenon in solid state physics. The Lorentz force affects electrons in such a way that all the system properties vary periodically with the inverse of the magnetic field[1]. Conventionally, QOs are observable at low temperatures $T$ and in absence of strong incoherence, and provide detailed information about the topology and shape of the Fermi surface.[1, 2] Yet, QOs are surprisingly ubiquitous. They also appear in non-Fermi liquids [3-5] and even in gapped systems such as Kondo insulators [6]. They were observed in graphite [7, 8], graphene [9, 10], organics [11], cuprates [12-14], perovskite heterostructures [15, 16], iron-pnictide superconductors [17], and moiré systems [18].

In moiré systems, huge superlattice spacing allows access to regime of large flux per unit cell $\Phi$. Precisely in this regime, recent experiments[19-22] have uncovered a new, peculiar type of QOs of conductivity: peaks at $\Phi$ equal to simple fractions of the flux quantum, i.e. $\Phi=\Phi_{0} p / q$ with $p, q$ coprime integers, and $p$ and $q$ small[21]. These BrownZak (BZ) oscillations are clearly distinct from the conventional Shubnikov-de Haas ( $\mathrm{SdH}$ ) oscillations: BZ QOs appear at elevated temperatures[20], and their frequency does not depend on the electron density $n$ (in 2D, SdH QOs have a frequency proportional to $n$ ). Some understanding of this phenomenon was reached by noting that the conductivity is high whenever the non-interacting density of states (DOS) consists of a small number $(q)$ of wide energy bands (magnetic "minibands")[20, 21]. States in wider bands should have a higher velocity, and therefore conduct better. However, this heuristic picture cannot explain the totality of experimental observations. In this paper we present a microscopic theory of conductivity in the Hubbard model and unexpectedly recover a phenomenology strikingly similar to that observed in the experiments of Refs. 20 and 21. Our analysis elucidates the essential role of incoherence for the $\mathrm{BZ}$ oscillations, and explains the temperature, doping and interaction trends in a systematic manner.

We employ the recently developed extension of the dynamical mean field theory (DMFT)[23] to finite magnetic fields [24-26]. In absence of the magnetic field, the DMFT solution of the Hubbard model was previously shown to describe the transport properties of various materials[2731] and cold atoms in optical lattices[32, 33]. The DMFT approximates the self-energy by a local quantity, and becomes exact in the limit of infinite coordination number. In a separate accompanying publication Ref. 26, we prove that the vertex corrections for the longitudinal conductivity cancel at the level of DMFT, regardless of the magnetic field (see also Refs. 34 and 25); this makes it possible to calculate conductivity by the Kubo bubble without any additional approximations. Our approach fully takes into account local correlations due to electron-electron (ee) interaction, and is formally applicable at any $T$, coupling strength $U$ and field $B$.

Our conductivity results exhibit oscillations that clearly correspond to the $\mathrm{BZ} \mathrm{QOs}$ observed in experiment. The oscillations have a frequency $p / q=1$ (corresponding to maxima at $p / q=1 / q$ ) and appear at relatively high $T$ where the $\mathrm{SdH}$ oscillations are getting thermally washed out. BZ either coexist with the $\mathrm{SdH}$ oscillations or appear as the sole oscillatory component. As $T$ is lowered, higher harmonics of BZ oscillations become more pronounced (peaks become sharper, and additional maxima at $p / q=$ $2 / q, 3 / q \ldots$ appear). Ultimately, at very low $T$, regular BZ oscillations give way to fractal behavior which does not yield any pronounced peaks in the Fourier spectrum. It turns out that the essential ingredient for the regular (sinusoidal) BZ oscillations are the incoherent electronic states. Incoherence allows for conduction processes that involve tunneling between two eigenstates of the Hamiltonian, and it is precisely the contribution of those procesess that oscilates at frequency $p / q=1$. Our numerical data suggests 

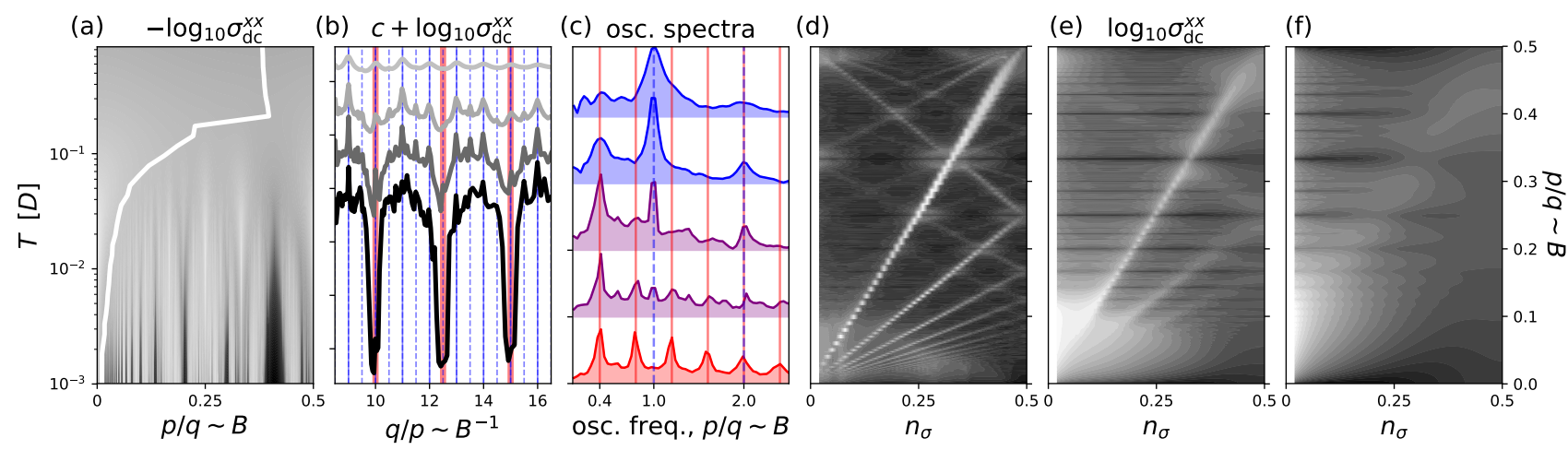

Figure 1. DMFT results for conductivity in the Hubbard model for $U=1 D$. (a) Conductivity as a function of temperature and field at band filling $n_{\sigma}=0.4$. Colorcode is logarithmic: black means $\log _{10} \sigma_{\mathrm{dc}}^{x x} \approx-7.95$, white means $\log _{10} \sigma_{\mathrm{dc}}^{x x} \approx 2.12$. White line: onset points of the non-monotonic behavior of $\left.\sigma_{\mathrm{dc}}^{x x}(B)\right|_{T}$. (b) Conductivity as a function of inverse magnetic field. Bottom to top: $T=0.0012,0.0049,0.0109,0.024 D$; lines are plotted on the log scale, and offset for the sake of clarity. (c) Frequency spectrum of conductivity in the range $p / q \in[0.03,0.15]$ at different temperatures. Bottom to top: $T=0.001,0.009,0.016,0.029,0.064 D$. Each spectrum is normalized to 1 and shifted for the sake of clarity. (b,c) Verical lines: peaks due to SdH oscillations (red), and BZ oscillations (blue). (d,e,f) Conductivity with respect to band filling and field at $T=0.005,0.03,0.1 D$, respectively. Colorcode: white means $-8.22,-3.57,-3.12$, black means $2.14,1.77,1.03$, respectively.

that in strongly correlated regimes, regular BZ oscillations should appear at very low temperature.

Model and method. We consider the Hubbard model on the square lattice with nearest-neighbor hopping $t$, coupling $U$ and band filling per spin $n_{\sigma}$, with $n=\sum_{\sigma} n_{\sigma}$. We use $D=4 t$ as the unit of energy. The field is included through Peierls phases for rational flux values $\Phi / \Phi_{0}=p / q$ to obtain commensurate magnetic cell [35]. We do not include the Zeeman term [36, 37], as it does not affect the QO frequencies, only their amplitudes[1]. We solve the problem within the DMFT with numerical renormalization group solver. Full details of our calculations are given in Ref. 26.

Results. Fig. 1(a) shows the conductivity for moderate doping and interaction $\left(n_{\sigma}=0.4, U=1\right)$ over a broad range of temperature and field (flux). At low $T$, we clearly see prominent oscillations. The onset of non-monotonic behavior is marked with the white line: it indicates the value of $B$ where the first extremum in $\sigma_{\mathrm{dc}}^{x x}$ is encountered for a given $T$. On Fig. 1(b) we zoom in on a narrow field range and plot $\sigma_{\mathrm{dc}}^{x x}$ as a function of $1 / B$ at several $T$. At low $T$, we see large dips in conductivity for $p / q=n_{\sigma} / i$ (red lines; $i$ is integer), corresponding to occurrences of a large gap in the density of states at the Fermi level. These are the SdH oscillations with a frequency related to the area of the Fermi sea $A_{\mathrm{FS}}$ by the Onsager relation $F=\Phi_{0} /(2 \pi)^{2} A_{\mathrm{FS}}, A_{\mathrm{FS}}=(2 \pi)^{2} n_{\sigma}$. In between the sharp SdH dips, one can observe a weak but highly nonmonotonous behavior of $\sigma_{d c}^{x x}$ with high-frequency oscillatory features exceeding the resolution of our calculations. With increasing $T$, the amplitude of the $\mathrm{SdH}$ oscillations is reduced in line with the Lifshitz-Kosevitch theory [2, 26], and the behavior in between the $\mathrm{SdH}$ dips becomes simpler: one gets spikes coinciding with small- $p /$ moderate- $q$ values of flux (denoted with blue lines: full line is $p=1$, dashed line is $p=2$ ). Ultimately, only regular sinusoidal oscillations of period 1 remain, with maxima at $p / q=1 / q$. Increasing $T$ further erases all non-monotonic behavior.

Fig. 1(c) shows the oscillation spectra obtained by Fourier transforming $\sigma_{\mathrm{dc}}^{x x}\left(B^{-1} \sim q / p\right)$ on the range $p / q \in$ $[0.03,0.15]$. At the lowest temperature we see strong peaks at $p / q=n_{\sigma}$ and its higher harmonics, corresponding to (sharp) SdH oscillations. The fractal behavior in between the SdH dips seen in Fig.1(b) does not produce a clear oscillatory signal[26]. As $T$ is increased, the peaks at $p / q=1$ and $p / q=2$ appear, while at the highest $T$ one is left only with the peak at $p / q=1$.

On Fig. 1(d,e,f) we plot the conductivity in the $\left(n_{\sigma}, B\right)$ plane. At low $T$, the $\mathrm{SdH}$ oscillation fans out from the $(0,0)$ point, clearly indicating the $n_{\sigma}$ dependence of the oscillation frequency. At a higher $T$, SdH oscillations become weaker; horizontal (i.e. $n_{\sigma}$-independent) stripes corresponding to fractal $\mathrm{BZ}$ oscillations become visible, and are particularly pronounced at small $p$ values. At the highest $T$ shown, only the BZ oscillations remain.

We summarize our observations by presenting in Fig. 2(a,b) the two relevant Hubbard model phase diagrams, showing the dominant type of (regular) oscillations, based on the Fourier spectrum of $\sigma_{\mathrm{dc}}^{x x}(q / p)$ in the field range $p / q \in[0.03,0.15]$. We also indicate the onset field for the non-monotonic behavior (grayscale colorcoding and the black contours). Clearly, the onset field depends strongly on $U$ and $n$; the non-monotonic behavior is stronger and requires less strong fileds in more coherent regimes (lower $U$ and/or higher doping away from halffilling $\delta=1-n$ ). Another notable trend is that the $\mathrm{BZ}$ oscillations start at a lower temperature in less coherent regimes (lower $\delta$ at fixed $U$; stronger $U$ at fixed $\delta$ ). 

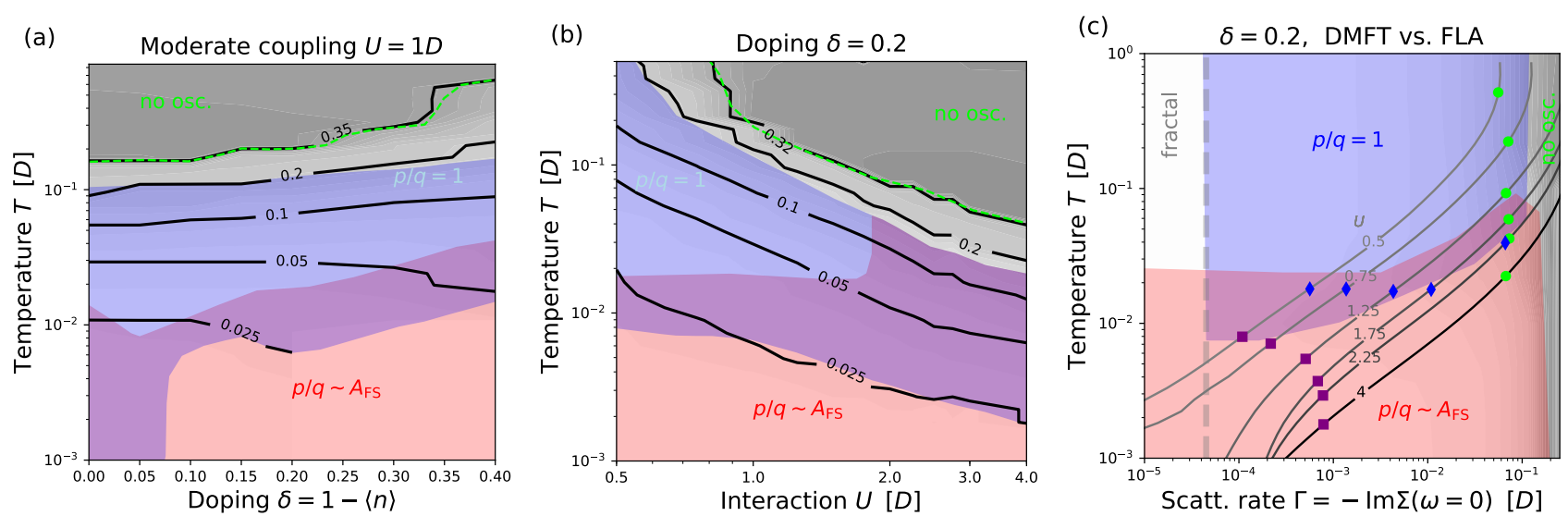

Figure 2. Phase diagrams showing the type of QOs observed in the range of field $p / q \in[0.03,0.15]$. (a) DMFT results in $(\delta, T)$ plane, (b) DMFT results in $(U, T)$ plane, (c) FLA results in $(\Gamma, T)$ plane. Red: SdH only. Purple: both SdH and BZ, but SdH dominant. Blue: BZ dominant $\left(p / q=1\right.$ peak stronger than $p / q \approx n_{\sigma}$ peak). Black shading and contours in $(\mathrm{a}, \mathrm{b})$ denote the value of the field where non-monotonic behavior starts in $1 /\left.\sigma_{\mathrm{dc}}^{x x}(B)\right|_{T}$ (analogous to the white line in Fig.1). Above the lime dashed line, no oscillations are detectable at any field strength. In (c), lines and symbols correspond to DMFT results, shading to FLA results. Lines are $\Gamma(T)$ for various values of $U$. Purple squares indicate where the BZ oscillations start with increasing $T$, blue diamonds where the BZ becomes dominant, and lime circles where all QOs cease (corresponding to the top edge of blue and purple regions in (b)).

To elucidate the role of incoherence we perform calculations within the the finite-lifetime approximation (FLA)[26], where lifetime of electronic states is set by hand by fixing the (local) self-energy to $\Sigma(\omega)=-i \Gamma$. We determine the phase diagram of FLA with respect to the two parameters of this toy model, the scattering rate $\Gamma$ and tempertaure $T$ (Fig. 2(c)). There appears to be a well defined upper cutoff value of $\Gamma$ for the observation of any QOs. For the observation of $\mathrm{SdH}$ oscillations, there is a relatively well defined upper cut-off $T$. The region of dominant regular BZ oscillations is additionally limited by lower cut-off $\Gamma$ and $T$. Below $\Gamma \approx 5 \times 10^{-5}$, fractal behavior is observed, with or without the $\mathrm{SdH}$ oscillations, depending on temperature. At moderate $\Gamma$, increasing the temperature alone does not wash out the BZ oscillations, and they persist up to infinite temperature.

We superimpose on the FLA phase diagram the DMFT results by identifying $\Gamma=-\operatorname{Im} \Sigma(\omega=0)$. In DMFT the self-energy has frequency dependence and depends on both $U$ and $T$. The grayscale lines represent the DMFT result for $\Gamma(T)$ for different $U$ values. The upper cut-off $\Gamma$ for QOs (lime points) holds in good agreement with FLA results, as well as the upper cut-off $T$ for $\mathrm{SdH}$ oscillations (blue diamonds). At low $U$, the lower cut-off $T$ for BZ oscillations is also in agreement with FLA. However, at high $U$, the discrepancy from FLA is significant: the sinusoidal BZ oscillations appear at much lower $T$ than one would expect based on a simple FLA toy model where $\Sigma$ has no frequency dependence. At very strong $U$, there rather seems to be a well defined lower cut-off $\Gamma$ for regular BZ QOs extending to very low $T$ (this lower $\Gamma$ cut-off being a bit higher than the one at high $T$ ). The observation of BZ oscillations at very low $T$ is therefore a clear indication of strong electronic correlations that go beyond simple incoherence effects.

Discussion. The trends related to incoherence and temperature can be understood from the linear-response transport theory underlying our calculations. The Kubo bubble for conductivity is illustrated in Fig. 3(a). At the level of the DMFT where the self-energy does not depend on the momentum, the product of two velocities $v_{\tilde{\mathbf{k}}, m, m^{\prime}} v_{\tilde{\mathbf{k}}, m^{\prime}, m}$ can be rewritten as a single factor with two kinetic-energy arguments, $v\left(\epsilon, \epsilon^{\prime}\right)$. Depending on temperature, effective scattering rate and chemical potential, different $\left(\epsilon, \epsilon^{\prime}\right)$ domains play a role [26]. In particular, only $\left(\epsilon, \epsilon^{\prime}\right)$ such that $\left|\epsilon-\epsilon^{\prime}\right|<\Gamma$ and $\epsilon^{(\prime)}-\mu<T$ give significant contributions. At low $T$, we observe that the $\mathrm{SdH}$ effect is already contained in $v\left(\epsilon, \epsilon^{\prime}\right)$. The oscillation spectrum for $v\left(\epsilon, \epsilon^{\prime} \approx \epsilon \approx \mu\right)$, exhibits a peak that moves with $\mu$ and coincides with $n_{\sigma}$. As the thermal window becomes larger, a wider range of $v\left(\epsilon, \epsilon^{\prime} \approx \epsilon\right)$ enter the calculation, yet oscillate with different frequencies, depending on $\epsilon$. This leads to dephasing and washing out of the $\mathrm{SdH}$ oscillations. By contrast, the $\mathrm{BZ}$ oscillation is mild at any given $\epsilon$, but it always has the same frequency $(p / q=1)$, thus its contribution accumulates with increasing $T$ and can become the dominant effect, as illustrated in Fig. 3(b). The domain of $v$ that turns out to oscillate with the BZ frequency is found at moderate $\left|\epsilon-\epsilon^{\prime}\right|$. Therefore, as the scattering rate $\Gamma$ is increased, those values enter the calculation and the $\mathrm{BZ}$ oscillations become visible in $\sigma_{\mathrm{dc}}^{x x}(q / p)$. The values of $v\left(\epsilon, \epsilon^{\prime}\right)$ at large $\left|\epsilon-\epsilon^{\prime}\right|$ do not oscillate with any particular frequency. As those get included at large $\Gamma$, all oscillations are ultimately overcome by the non-oscillatory contributions, as illustrated in Fig. 3(c). The velocity $v$ is the only source of BZ oscillations in the Kubo bubble, as the 
a)



b)

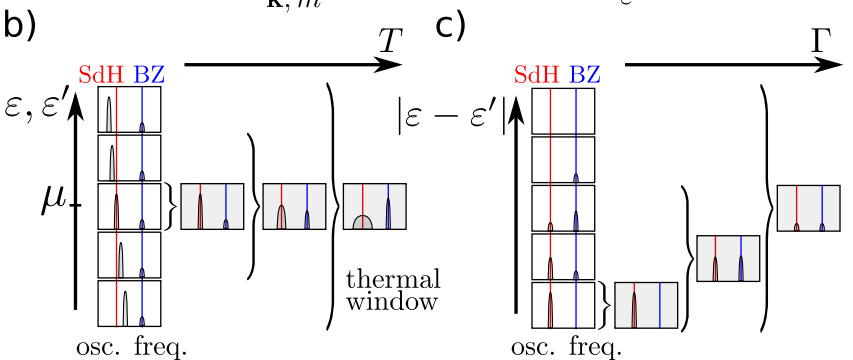

d)

FLA $, \mu=-0.1 D, \sigma_{\mathrm{DC}}^{\times x}$ contributions

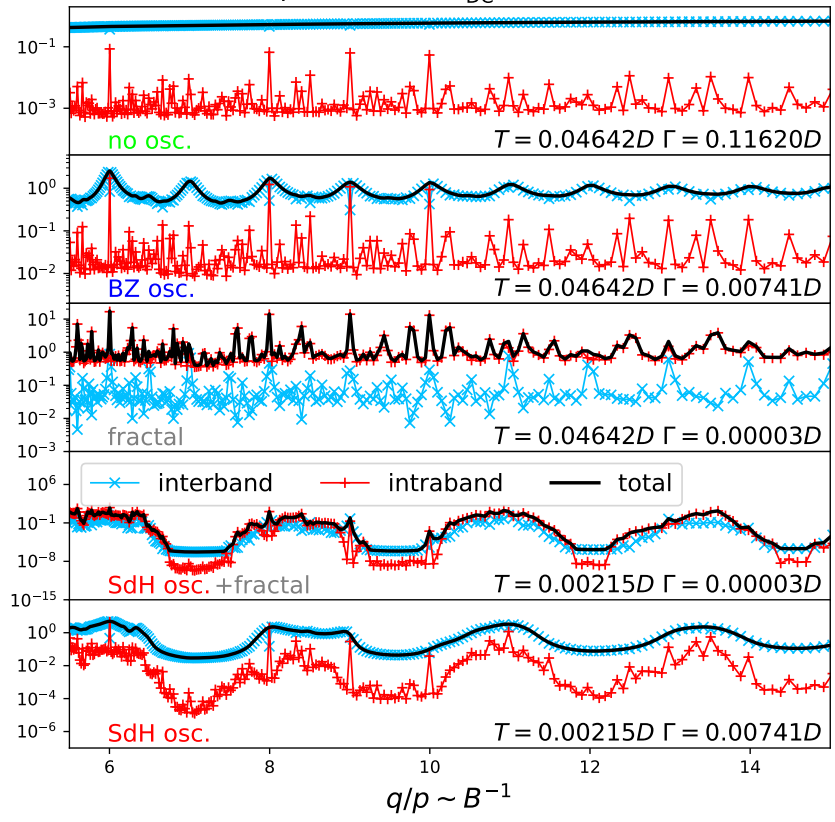

Figure 3. (a) Diagrammatic representation of the Kubo bubble. Left: in general; right: at the level of the DMFT. $(\tilde{\mathbf{k}}, m)$ denotes eigenstates of the non-interacting Hamiltonian (see [26] for details). Red/lime triangles are the velocity vertices, in DMFT rewritten as a single factor depending on two kinetic energies, $v\left(\varepsilon, \varepsilon^{\prime}\right)$. (b,c) White panels: Oscillation spectra of $v\left(\varepsilon, \varepsilon^{\prime}\right)$ at a given $\left(\varepsilon, \varepsilon^{\prime}\right)$. Gray panels: Oscillation spectra of $v$ integrated over the relevant $\left(\varepsilon, \varepsilon^{\prime}\right)$-domain, depending on model parameters ( $T$ and $\Gamma$ ), as indicated by the large curly bracket; (b) Trend with respect to temperature. (c) Trend with respect to the scattering rate. (d) Field dependence of conductivity and the contributions of interband $\left(\epsilon \neq \epsilon^{\prime}\right)$ and intraband $\left(\epsilon \approx \epsilon^{\prime}\right)$ processes in FLA in four different parameter regimes.

Green's function and the self-energy do not have an oscillatory component at the frequency of BZ oscillations[26].

In previous works[20, 21], the BZ oscillations were connected with the velocity of the magnetic minibands, calculated as $v=\partial \epsilon_{\tilde{\mathbf{k}}, m} / \partial \tilde{k}_{x}$. Nevertheless, it is important to note that the eigenstates of the non-interacting Hamiltonian do not have a well defined velocity in the presence of the field. Rather, the velocity $v_{\tilde{\mathbf{k}}, m, m^{\prime}}$ is $a m a$ - trix in the miniband space $m, m^{\prime}$. In previous works this was not taken into account and the results were interpreted in terms of only the intraband processes (diagonal elements of $v$ ). This would be well justified only in the limit of coherent, long-lived quasiparticle states. However, increasing $T$ even at weak coupling leads to decoherence of electron states, which activates the contribution of offdiagonal velocity components and even makes them fully dominant[26]. This corresponds to $m \neq m^{\prime}$ (or $\varepsilon \neq \varepsilon^{\prime}$ ) terms in the Kubo bubble in Fig.3(a). For these interband processes, the amplitude is determined by the the probability of tunneling between two minibands upon measurement of velocity. We illustrate the relative contributions of interband and intraband processes to overall dc conductivity on Fig. 3(d) in 5 different regions of parameters of the FLA toy model. These plots reveal that the diagonal components of the velocity cannot account for the regular sinusoidal BZ oscillations, but only for the fractal behavior that is observed at low $\Gamma$. It is interesting to note that even at very high $\Gamma$, the intraband processes still exhibit strong fractal behavior, while the overall conductivity is already devoid of any apparent QOs. This indicates that the regular BZ oscillations are not a simple "smoothing" of the fractal behavior due to widened peaks in the (fractal) spectral function. Rather, this is a separate phenomenon, ultimately due to oscillations in the tunneling amplitudes $v_{\tilde{\mathbf{k}}, m, m^{\prime} \neq m}$.

Relation to experiment. Both the fractal behavior (peaks in $\sigma_{\mathrm{dc}}^{x x}$ up to $p / q=4 / q$ ) and the regular BZ oscillations have been observed in experiment[20,21]. The $T$-trend observed in Fig. 1(d,e,f) is in qualitative agreement with the experimental findings of Ref. 20. Note that the lattice in this moire system is different from that in our model, and that the dominant interaction in graphene at high $T$ is likely of the electron-phonon (e-ph) type, while our Hamiltonian only includes e-e repulsion. The agreement despite such differences indicates a significant level of universality in these phenomena. Notwithstanding, the doping trend at the highest temperature is in apparent contrast to the measurements in Ref. 20. In our Fig. 1(f), BZ oscillations are regular (sinusoidal) close to half-filling; closer to the empty band limit a stronger fractal behavior remains in place. In the corresponding high- $T$ experimental result in Ref. 20 (Fig.2B,C), only the regular oscillations are observed, and no oscillations at all are observed close to the "neutrality point" (corresponding to the empty band limit in our calculations). This discrepancy appears to be due to the difference in the scattering mechanism: the e-e scattering rate goes to zero as the band empties, but the e-ph scattering rate does not. The FLA calculation[26] where $\Gamma$ is fixed regardless of the doping clearly reproduces the doping-trend observed in the experiment. Similarly, at low temperature in the Hubbard model, one observes both the SdH oscillations and fractal behavior (Fig. 1(b)); In experiment, there are cases where only $\mathrm{SdH}$ oscillations are observed at low temperature. This discrepancy is, again, likely due to the difference in scattering mechanisms. In the Hubbard model 
the scattering rate goes down with temperature (Fig.2(c)). If the scattering rate is kept fixed at a moderate value (as in FLA), at low $T$ one only observes the SdH effect (see bottom panel in Fig. 3(d)).

Conclusion. We have studied the magnetic quantum oscillations of longitudinal DC conductivity in the 2D Hubbard model. We observe three types of non-monotonic behavior in $\sigma_{\mathrm{dc}}^{x x}$ : 1) Shubnikov-de Haas oscillations with frequency $p / q=n_{\sigma}$ (and higher harmonics), at low temperature; 2) fractal behavior of conductivity with peaks at $\Phi / \Phi_{0}=1 / q, 2 / q, 3 / q \ldots$, in the coherent regimes; 3 ) sinusoidal $p / q=1$-frequency oscillations, in moderately incoherent regimes (the Brown-Zak oscillations, BZ). Our findings are in striking agreement with recent experimens on graphene superlattices. The discrepancies from experiment can be traced back to a difference in interactions present in the system. The oscillation phenomenology crucially depends on the scattering rate, and can thus be used in experiment as a characterization tool for scattering mechanisms. The fractal behavior is ultimately a manifestation of the Hofstadter butterfly, and is an indication of a low scattering rate; in contrast, the $\mathrm{BZ}$ oscillations indicate a higher scattering rate, and when observed at very low temperature are an indication of a strong e-e coupling. Our results present clear predictions for future experiments where the dependence on coupling strength and doping might be investigated.

Computations were performed on the PARADOX supercomputing facility (Scientific Computing Laboratory, Center for the Study of Complex Systems, Institute of Physics Belgrade). J. V. acknowledges funding provided by the Institute of Physics Belgrade, through the grant by the Ministry of Education, Science, and Technological Development of the Republic of Serbia, as well as by the Science Fund of the Republic of Serbia, under the Key2SM project (PROMIS program, Grant No. 6066160). R. Ž. is supported by the Slovenian Research Agency (ARRS) under Program P1-0044 and Projects J1-1696 and J1-2458.

[1] D. Schoenberg, Magnetic oscillations in metals (Cambridge Univ. Press, Cambridge, UK, 1984).

[2] I. M. Lifshitz and A. M. Kosevich, Sov. Phys. JETP 2, 636 (1956).

[3] F. Denef, S. A. Hartnoll, and S. Sachdev, Phys. Rev. D 80, 126016 (2009).

[4] S. A. Hartnoll and D. M. Hofman, Phys. Rev. B 81, 155125 (2010).

[5] D. V. Else, R. Thorngren, and T. Senthil, Phys. Rev. X 11, 021005 (2021).

[6] J. Knolle and N. R. Cooper, Phys. Rev. Lett. 115, 146401 (2015).

[7] D. E. Soule, J. W. McClure, and L. B. Smith, Physical Review 134, A453 (1964).

[8] S. B. Hubbard, T. J. Kershaw, A. Usher, A. K. Savchenko, and A. Shytov, Phys. Rev. B 83, 035122 (2011).

[9] K. S. Novoselov, A. K. Geim, S. V. Morozov, D. Jiang, M. I. Katsnelson, I. V. Grigorieva, S. V. Dubonos, and A. A. Firsov, Nature 438, 197 (2005).

[10] Y. Zhang, Y.-W. Tan, H. L. Stormer, and P. Kim, Nature 438, 201 (2005).

[11] M. V. Kartsovniuk and V. G. Peschansky, Low Temperature Physics 31, 185 (2005).

[12] N. Doiron-Leyraud, C. Proust, D. LeBoeuf, J. Levallois, J.B. Bonnemaison, R. Liang, D. A. Bonn, W. N. Hardy, and L. Taillefer, Nature 447, 565 (2007).

[13] S. E. Sebastian, N. Harrison, E. Palm, T. P. Murphy, C. H. Mielke, R. Liang, D. A. Bonn, W. N. Hardy, and G. G. Lonzarich, Nature 454, 200 (2008).

[14] S. E. Sebastian and C. Proust, Annual Review of Condensed Matter Physics 6, 411 (2015).

[15] A. D. Caviglia, S. Gariglio, C. Cancellieri, B. Sacépé, A. Fête, N. Reyren, M. Gabay, A. F. Morpurgo, and J.-M. Triscone, Phys. Rev. Lett. 105, 236802 (2010).

[16] P. Moetakef, D. G. Ouellette, J. R. Williams, S. J. Allen, L. Balents, D. Goldhaber-Gordon, and S. Stemmer, Applied Physics Letters 101, 151604 (2012).

[17] A. Carrington, Reports on Progress in Physics 74, 124507 (2011).

[18] Y. Cao, J. Y. Luo, V. Fatemi, S. Fang, J. D. SanchezYamagishi, K. Watanabe, T. Taniguchi, E. Kaxiras, and P. Jarillo-Herrero, Phys. Rev. Lett. 117, 116804 (2016).

[19] B. Hunt, J. D. Sanchez-Yamagishi, A. F. Young, M. Yankowitz, B. J. LeRoy, K. Watanabe, T. Taniguchi, P. Moon, M. Koshino, P. Jarillo-Herrero, and R. C. Ashoori, Science 340, 1427 (2013).

[20] R. K. Kumar, X. Chen, G. H. Auton, A. Mishchenko, D. A. Bandurin, S. V. Morozov, Y. Cao, E. Khestanova, M. B. Shalom, A. V. Kretinin, K. S. Novoselov, L. Eaves, I. V. Grigorieva, L. A. Ponomarenko, V. I. Fal'ko, and A. K. Geim, Science 357, 181 (2017).

[21] R. K. Kumar, A. Mishchenko, X. Chen, S. Pezzini, G. H. Auton, L. A. Ponomarenko, U. Zeitler, L. Eaves, V. I. Fal'ko, and A. K. Geim, Proceedings of the National Academy of Sciences 115, 5135 (2018).

[22] J. Barrier, P. Kumaravadivel, R. K. Kumar, L. A. Ponomarenko, N. Xin, M. Holwill, C. Mullan, M. Kim, R. V. Gorbachev, M. D. Thompson, J. R. Prance, T. Taniguchi, K. Watanabe, I. V. Grigorieva, K. S. Novoselov, A. Mishchenko, V. I. Fal'ko, A. K. Geim, and A. I. Berdyugin, Nature Communications 11, 5756 (2020).

[23] A. Georges, G. Kotliar, W. Krauth, and M. J. Rozenberg, Rev. Mod. Phys. 68, 13 (1996).

[24] S. Acheche, L.-F. Arsenault, and A.-M. S. Tremblay, Phys. Rev. B 96, 235135 (2017).

[25] A. A. Markov, G. Rohringer, and A. N. Rubtsov, Phys. Rev. B 100, 115102 (2019).

[26] J. Vučičević and R. Žitko, "Conductivity in the hubbard model: orbital effects of magnetic field," (2021), arXiv:2104.14578.

[27] P. Limelette, P. Wzietek, S. Florens, A. Georges, T. A. Costi, C. Pasquier, D. Jérome, C. Mézière, and P. Batail, Phys. Rev. Lett. 91, 016401 (2003).

[28] H. Terletska, J. Vučičević, D. Tanasković, and V. Dobrosavljević, Phys. Rev. Lett. 107, 026401 (2011).

[29] J. Vučičević, H. Terletska, D. Tanasković, and V. Do- 
brosavljević, Phys. Rev. B 88, 075143 (2013).

[30] T. Furukawa, K. Miyagawa, H. Taniguchi, R. Kato, and K. Kanoda, Nature Physics 11, 221 (2015).

[31] J. Vučičević, D. Tanasković, M. J. Rozenberg, and V. Dobrosavljević, Phys. Rev. Lett. 114, 246402 (2015).

[32] J. Vučičević, J. Kokalj, R. Žitko, N. Wentzell, D. Tanasković, and J. Mravlje, Phys. Rev. Lett. 123, 036601 (2019).
[33] P. T. Brown, D. Mitra, E. Guardado-Sanchez, R. Nourafkan, A. Reymbaut, C.-D. Hébert, S. Bergeron, A.-M. S. Tremblay, J. Kokalj, D. A. Huse, P. Schauß, and W. S. Bakr, Science 363, 379 (2018).

[34] A. Khurana, Phys. Rev. Lett. 64, 1990 (1990).

[35] D. R. Hofstadter, Physical Review B 14, 2239 (1976).

[36] L. Laloux, A. Georges, and W. Krauth, Phys. Rev. B 50, 3092 (1994).

[37] J. Bauer and A. C. Hewson, Phys. Rev. B 76, 035118 (2007). 